\title{
FETAL PHAGOCYTES LOADED WITH HPV E7 PEPTIDES COULD ELICIT CYTOTOXICITY AGAINST TC-I CELLS
}

\author{
Jeng-Chang Chen, MD, PhD' and Liang-Shiou Ou, MD²
}

\begin{abstract}
'Department of Surgery, Chang Gung Children's Hospital, College of Medicine, Chang Gung University, Taiwan ${ }^{2}$ Division of Allergy, Asthma and Rheumatology, Department of Pediatrics, Chang Gung Children's Hospital, College of Medicine, Chang Gung University, Taiwan
\end{abstract}

\section{Background and aims:}

In utero exposure to ovalbumin caused Th2 sensitization to ovalbumin. This adaptive immunity was initiated by fetal phagocytes that internalized ovalbumin, attenuated its proteolysis and then differentiated towards dendritic cells for delayed presentation. This study was aimed to examine whether fetal phagocytes could elicit Th1 cytotoxicity against tumor cells.

\section{Methods:}

Fetal phagocytes from gestational day 14 C57BL/6 fetal liver and peritoneal cavity were pulsed with human papillomavirus (HPV) E7 peptides overnight and examined by immunofluorescence staining and flow cytometry. Then, they were transferred intraperitoneally into 4-6 week-old C57BL/6 mice. Four-six weeks later, recipients were examined for serum anti-E7 lgG levels

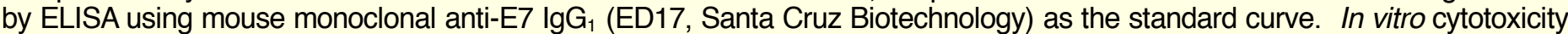
of recipients' splenic lymphocytes to TC-1 cells was evaluated by 4',6-diamidino-2-phenylindole (DAPI) viability dye and lactate dehydrogenase $(\mathrm{LDH})$ release from lysed TC-1 cells.

\section{Results:}
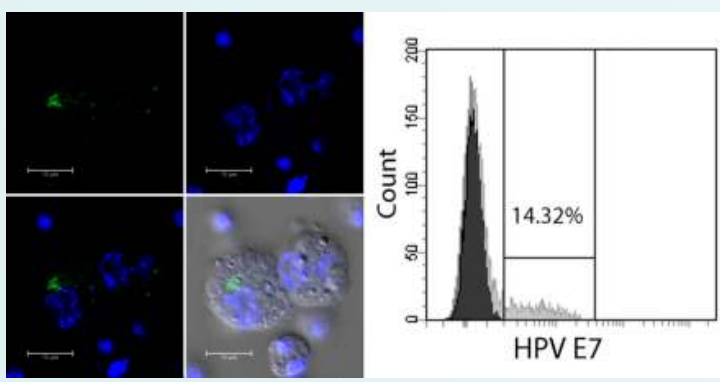

HPVE7

Figure 1. Endocytosis of HPV E7 by fetal macrophages. Cells harvested from fetal peritoneum or liver were pulsed with HPV E7 overnight, and then subjected to immunofluorescence staining by mouse anti-E7 $\lg _{1}$ and FITCconjugated anti-mouse IgG secondary antibody, followed by DAPI nuclear counterstain. Cells were inspected under a confocal microscope and analyzed by flow cytometry. HPV E7 was detected within the cytoplasm of fetal macrophages that contained several intracellular vacuoles. The histogram obtained from a representative peritoneum sample showed that $14.32 \%$ of cells phagocytosed HPV E7 following overnight coculture with HPV E7.

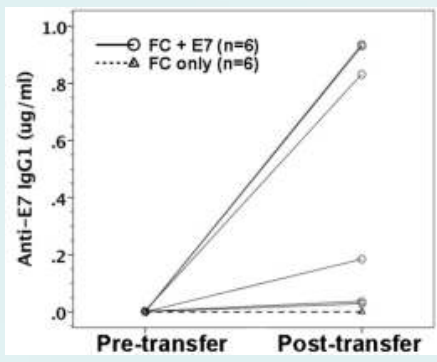

Figure 2. The generation of anti-E7 IgG $_{1}$ following adoptive transfer of HPV E7-loaded fetal phagocytes. Gestational day 14 murine fetal phagocytes were loaded with HPV E7 peptides and then transferred into adult mice. It successfully induced the generation of antiE7 lgG $1(P=0.045)$. The control mice were subjected to adoptive transfer of fetal phagocytes without the pulse of E7 $(P=0.175)$. Coupled circles or triangles represent paired data from an individual mouse before and after adoptive transfer of fetal phagocytes with or without the pulse of HPV E7 peptides.

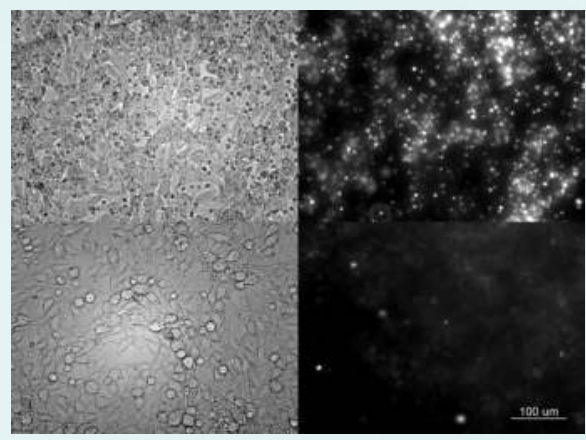

Figure 3. Viability assay of TC-1 cells. C57BL/6 mice were subjected to adoptive transfer of HPV E7-loaded C57BL/6 fetal phagocytes. Following restimulated with HPV E7 peptides, their splenic lymphocytes were added to TC-1 cells grown in culture plates and incubated overnight. TC-1 cells were observed under a fluorescence microscope following the treatment of DAPI viability dye. There were numerous small nucleated lymphocytes (phase contrast fields, upper left panel) and areas of TC-1 cells with bright fluorescence-stained large nuclei (upper right panel), indicative of nonviable or dying TC-1 cells. The controls were C57BL/6 mice that only received the transfer of $\mathrm{C} 57 \mathrm{BL} / 6$ fetal phagocytes, showing few small nucleated lymphocytes and vey dim fluorescence (lower panels).

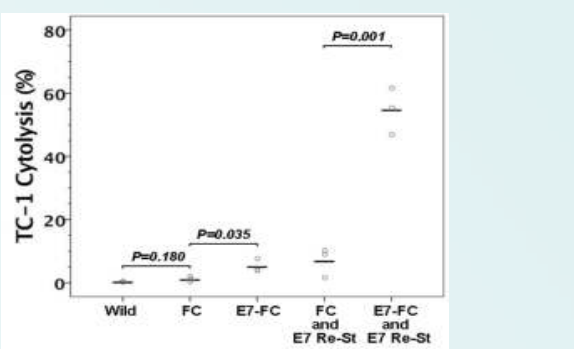

Figure 4. Quantitative assay for cell-mediated cytotoxicity against TC-1 cells. C57BL/6 mice were subjected to adoptive transfer of C57BL/6 fetal phagocytes with (E7-FC) or without (FC) HPV E7 loading. The cytotoxicity of their lymphocytes against TC-1 cells were quantified in vitro by LDH release in cell culture supernatants. E7-FC showed a higher TC-1 lysis than FC and wild type. Re-stimulated with HPV E7 (E7 ReSt), lymphocytes of E7-FC obtained an markedly increased capacity of TC-1 lysis, as compared with those of FC.

\section{Conclusion:}

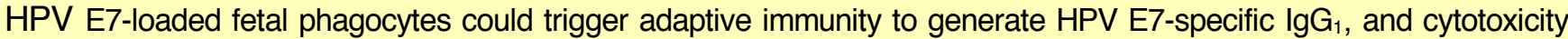
against TC-1 tumor cells. It cast light on the importance of fetal phagocytes for initiating cytotoxicity as a tumor immune surveillance system to eliminate embryogenesis-derived malignant-transformed cells within normal repertoire of embryo development. 